\title{
ORIGINAL EFFECTIVE, SAFE TECHNIQUE OF OBTAINING PLATELET RICH PLASMA BY CENTRIFUGATION OF THE BLOOD PLASMA IN MODIFIED SYRINGE CONTAINERS
}

\author{
Sergiy Chetverikov \\ Surgery department No. 4 with oncology course \\ Odessa National Medical University \\ 2 Valichovskiy lane, Oddesa, Ukraine, 65082 \\ dmitriyatanasov@gmail.com \\ Dmitro Atanasov \\ Surgery department No. 4 with oncology course \\ Odessa National Medical University \\ 2 Valichovskiy lane, Oddesa, Ukraine, 65082 \\ dmitriyatanasov@gmail.com
}

\begin{abstract}
The aim: to develop, substantiate an effective and safe technology for producing PRP (platelet rich plasma). To quantify the substrate based on the recommended centrifugation protocols.

Materials and methods: the effectiveness of the original harvesting protocol was evaluated by quantifying the number of platelets. The proposed technique is formed basing on the basic principles of double centrifugation of whole blood in test tubes with anticoagulant, separation with the release of a plasma layer with a high content of platelets.

The centrifuging mode for quantifying the effectiveness of the substrate was selected according to recommendations based on a study confirming maximum efficiency $(160 \mathrm{~g} \times 10 \mathrm{~min}+250 \mathrm{~g} \times 15 \mathrm{~min})$.

For quantitative evaluation, blood was collected from 10 healthy volunteers ( 7 men, 3 women) with an average age of 26.0 \pm 2.6 , and centrifuged in standard mode. Quantitative evaluation of platelets of whole blood and the obtained PRP substrate was carried out with a semi-automatic analyzer.

Results: the proposed technique is based on the use as a container for centrifuging a syringe with a LuerLock design, which is hermetically sealed with a congruent plug, adapted by the external size of the centrifuge rotor bowl. Phase selection after centrifugation was performed by aspiration of the syringe contents after centrifugation is performed through a three-way valve. The substrate was obtained by repeated centrifugation of the contents, which allows obtaining a variable volume and platelet concentration in PRP. The amount of platelets (PLT) of whole blood is 227.0 \pm 57.0 thousand per ml. PLT PRP $945.0 \pm 279.0$ thousand per $\mathrm{ml}$.

Conclusions: the proposed method of separation of whole blood with the release of the platelet rich plasma demonstrates high efficiency, which corresponds to the level of increasing the number of platelets in reducing the volume at the level of the best ready-made solutions.

The equipment is economical and does not require highly specialized equipment and consumables. The proposed technique provides a wide choice to the performer in the received volume of the substrate.
\end{abstract}

Keywords: platelet rich plasma, platelets, red blood cells, centrifugation, syringe, Discofix, combi-stopper, harvesting.

\section{Introduction}

At the present stage of development of medicine, platelet rich plasma (PRP) - based plasma products, that have found application and have become firmly established in clinical practice in many medical fields $[1,2]$. The stimulating effect on post-traumatic, as well as age-related skin changes ensured the popularity and widespread use of PRP in reconstructive, aesthetic surgery, and cosmetology $[3,4]$. Most of the thematic publications prove the high effectiveness of PRP in surgical dentistry, orthopedics $[4,5]$. In addition, a significant number of publications use PRP in the practice of gynecologists, urologists, cardiovascular surgeons [6, 7].

The overwhelming majority of authors state the safety and high efficiency of the techniques. Given that the substrate is autologous, subject to the technology of obtaining leveled the risk of allergic, infectious complications. The safety of the substrate has been proven in the publications of 
many authors $[8,9]$. However, the effectiveness according to different authors varies significantly and depends on the quantitative characteristics, the main and affordable rapid assessment of which is the number of platelets per unit volume $[10,11]$. This indicator determines the compliance of PRP with standardized criteria as well as the expected clinical effect. There are various PRP production systems on the market. In some cases, the figures declared by manufacturers have not been confirmed in practice [12]. In all cases, whole blood in a container with an anticoagulant is subjected to centrifugation with phase separation and subsequent separation of red blood cells and part of the plasma volume $[12,13]$. Separation of whole blood is achieved by obtaining plasma with an increased number of platelets. Fundamental differences were noted in the design of containers for sampling, centrifugation, as well as the technology of selection of the separated phases. The most common option is simple tubes, standard forms of the type PRGF EndoRet (Plasma Rich in Growth Factors) from the Biotechnology Institute, Spain, the main drawback of which is the manual selection with a syringe, which requires certain skills in performance [12]. The kits containing the helium separator of fractions of the RegenLab type (Switzerland) have the disadvantages of high complexity, high cost of the kit, as well as significant losses in platelet count, which is determined by the separation technology itself $[12,14]$. Other systems for obtaining PRP differing in the design of the container with a more complex device, allowing the selection of separated phases to be performed according to the standard procedure are less demanding on the skills of the contractor, but they are expensive $[15,16]$. Thus, it is difficult to isolate the optimal variant of the PRP production system taking into account the need for volume, as well as the substantial cost of centrifuges and consumable sets for most systems $[15,17]$.

\section{Aim of the work}

Develop a technology for obtaining PRP with a safe and highly efficient procedure that allows obtaining a variable volume of the substrate using available consumables that will provide high economic efficiency.

\section{Materials and methods}

The results of the assessment of quantitative indicators of compliance of the substrate of plasma rich with platelets obtained by the original method of compliance with standard requirements were analyzed.

The proposed technique are basing on the basic principles of dual centrifugation of whole blood in test tubes with an anticoagulant, separation with the release of a plasma layer with a high content of platelets.

In a patient by venipuncture of the peripheral or central vein, blood is collected in a syringe with a LuerLock attachment mechanism filled in $10 \%$ of the volume with a solution of the anticoagulant CITRAT glucose solution A (ACD-A) and filled to the full volume with the patient's blood after which the syringe is sealed under aseptic conditions tightly and congruently to LuerLock stub CombiStopper. Refinement of the shape of the container syringe was carried out sharply and did not require compliance with the strict conditions of asepsis due to the fact that the system was closed during these stages of harvesting. The selection of centrifuged phases was carried out through a three-way tap of Discofix $\mathrm{C}$ in compliance with the aseptic conditions.

In previously published studies, the maximum efficiency of platelet concentration was demonstrated in double centrifugation with a $160 \mathrm{~g} \times 10 \mathrm{~min}+250 \mathrm{~g} \times 15 \mathrm{~min}$ mode [18]. In choosing the centrifugation protocol, they relied on these indicators, adapting the rate of acceleration to the rotor design of the centrifuge used. A subjective assessment of the separation of the buffer layer was performed based on the nature of the distribution of the phases in standard tubes with similar conditions.

The clinical stage of the work was performed in the conditions of the surgical department of the Center for Reconstructive Restorative Medicine and the Department of Surgery No. 4 of the ONMEDU.

For quantitative evaluation, blood was collected from 10 healthy volunteers ( 7 men, 3 women) with an average age of $26 \pm 2.6$ years, centrifuged in a Hettich Rotorfix 32A centrifuge (Ger- 
many) with a rotor adapted for Arthrex ACP containers. Estimation of the platelet count of whole blood and the obtained PRP substrate was estimated using a Micro-CC 20 semi-automatic hematology analyzer (HTI USA). Statistical processing was performed using standard MS Office Excel 2013 algorithms. The confidence interval was determined by calculating Student's t test $(\mathrm{p}<0.05)$.

\section{Results}

Based on the biological inertness and availability of congruent components, we determined the syringes with the connector of the LuerLock design as the optimal container for centrifuging.

Formulated protocol:

In a patient by venipuncture of the peripheral or central vein, blood is collected in a syringe with a LuerLock attachment mechanism filled in $10 \%$ of the volume with a solution of the anticoagulant CITRAT glucose solution A (ACD-A) and filled to the full volume with the patient's blood after which the syringe is sealed under aseptic conditions tightly and congruently to LuerLock stub CombiStoper.

In order to reduce the size of the "syringe container", it is proposed to remove the excess length of the piston with the formation of a uniformly flat surface on which the container will be supported during the centrifugation process. Removing excess piston length does not require aseptic conditions and can be done with a sharp scalpel or forceps. In order to adapt the diameter of the "syringe container", it is additionally necessary in a similar way to remove the excess surface of the finger rest.

By the method described above, the patient's blood was collected. In order to reduce the size of the "syringe container", it has been proposed to remove the excess length of the piston with the formation of a uniformly flat surface upon which the container will be supported during the centrifugation process (Fig. 1). Removing excess piston length does not require aseptic conditions and can be done with a sharp scalpel or forceps. In order to adapt the diameter of the "syringe container", it is additionally necessary in a similar way to remove the excess surface of the finger rest (Fig. 1).

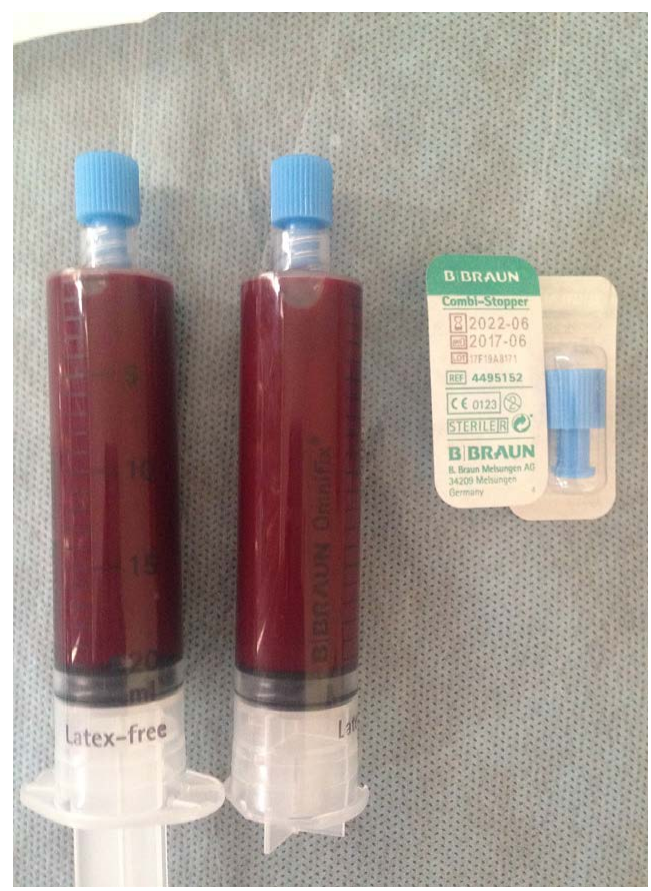

Fig. 1. Type of adaptation of the external dimensions of the syringe after the material is taken, the combi-stopper closes

The resulting "syringe container" should receive the dimensions corresponding to the internal dimensions of the centrifuge container used in this case. Thus, for most centrifuges used in laboratory and clinical practice, it is advisable to use syringes of $10 \mathrm{ml}$, in some cases when using 
rotors of centrifuges with large-diameter bowls, it is advisable to use syringes of $20 \mathrm{ml}$. The limiting conditions for the selection of centrifuges is the possibility of observing a controlled rotational speed in the range of $100-250 \mathrm{~g}$ and the presence of a horizontal rotor with freely deflected cups. After placing the "syringe containers" in the rotor cups installed in the centrifuge, centrifuging is performed in pairs with an acceleration of $160 \mathrm{~g}$ in the range from 12 to $16 \mathrm{~min}$, the so-called "soft spin" $[12,18]$. Time may vary depending on the conditions of the procedure (hematocrit level, which was determined before, temperature and humidity of the air in the room). After the first centrifugation, the "syringe containers" are carefully removed and placed in a stand vertically.

The result of the first stage of centrifugation is blood distribution with separation of the erythrocyte fraction deposited from the side of the syringe piston, the blood plasma remains superficially in the container and the thrombolic leukocyte layer located in the buffer zone between the erythrocyte layer and plasma and under the correct mode of centrifugation conditions selection looks gradiently over $20 \%$ of the total height of the plasma column. After the combi-stopper is removed, the corresponding end of the traveling crane Discofix $\mathrm{C}$ is attached to each of the syringes while maintaining a strictly vertical orientation in the LuerLock connector. The rotary mechanism of Discofix is set to the position of blocking free connector and combines both syringes with each other. An empty syringe should be installed with the piston at 0 .

Carrying out the movement of the piston of an empty syringe "to itself" by a controlled movement, the plasma layer is aspirated together with the buffer layer through a three-way valve (Fig. 2). This procedure is performed with each on the use of syringes alternately, with the appropriate addition of a syringe aspiration of plasma with a buffer layer to the full volume. Syringes are closed with combi-stopper plugs. For example: using 4 syringes with a volume of $20 \mathrm{ml}$ at the first stage of centrifugation, plasma with a buffer layer in each syringe will take approximately $11 \mathrm{ml}$, that is, from 4 syringes it can be taken in 2 syringes $20 \mathrm{ml}$ used "syringe containers" with erythrocyte layer and combi-stoppers are disposed of.

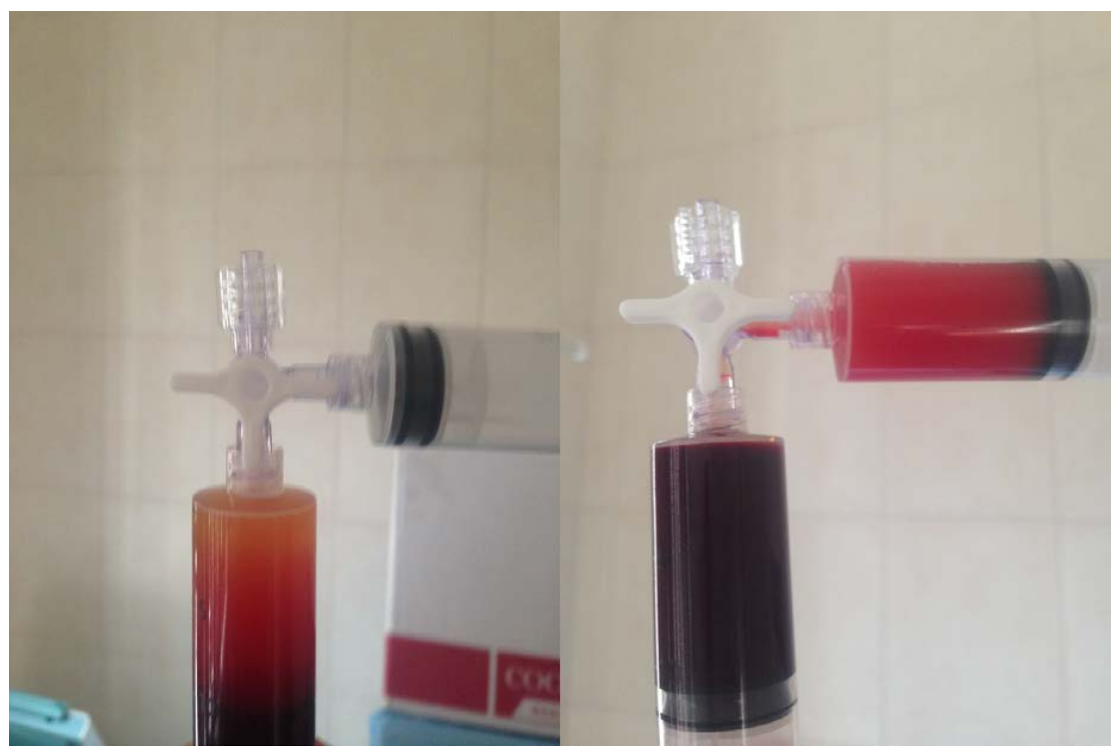

Fig. 2. The separation of the erythrocyte layer. Selection by aspiration of the separated layer: plasma + buffer layer

It is important at this stage to maintain the level of aspiration with the maximum approximation to the erythrocyte layer. The need for manual control of the level of aspiration is one of the key disadvantages of this method of separation of blood components. This disadvantage can be partially eliminated due to the use of a controlling mechanism with a free piston moving along the thread with a wide pitch in the coupling that is attached to the body of the "syringe container". By moving the movable piston along the thread, a well-controlled, metered advancement of the piston of the "syringe container" is performed, which allows a more precise determination of the level of 
separation of the erythrocyte layer and reduces the risk of excessive red blood cell or insufficient collection of the buffer layer. Having obtained syringes in which plasma with a buffer layer is selected, they are followed by a similar procedure - removing the excess length of the piston and the finger stops, providing the shape of the centrifuge rotor adapted to the internal size of the rotor container. Syringes containers are centrifuged in pairs. The purpose of the second stage of centrifugation is the concentration of the platelet layer.

Centrifugation is performed with a high acceleration of $-250 \mathrm{~g}$ for $-10-15$ minutes - the so-called "hard spin". It is possible to place "syringe containers" vertically in a centrifuge bowl a combi-stopper - then, as a result of centrifugation, the plasma fraction enriched with platelets will occupy one third of the volume on the piston side (Fig. 3). It is necessary to aspirate $2 / 3$ of the so-called "poor platelet plasma" through the Diskix, this plasma is not used. 1/3 of the remaining volume is plasma rich with platelets. Similarly, through a three-way valve, platelet-rich plasma is aspirated into a syringe of the LuerLock design. In order to activate platelet-enriched plasma, it is advisable to use a widely available $\mathrm{CaCl}_{2}$ solution of $10 \%$ in a ratio of $10 \%$ of the total plasma volume of platelet-rich as the activating agent [20]. The volume, administration of the activator, as well as the choice of another activating agent, may vary depending on the potential application and the need for exposure or long-term use of the drug platelet-rich plasma.

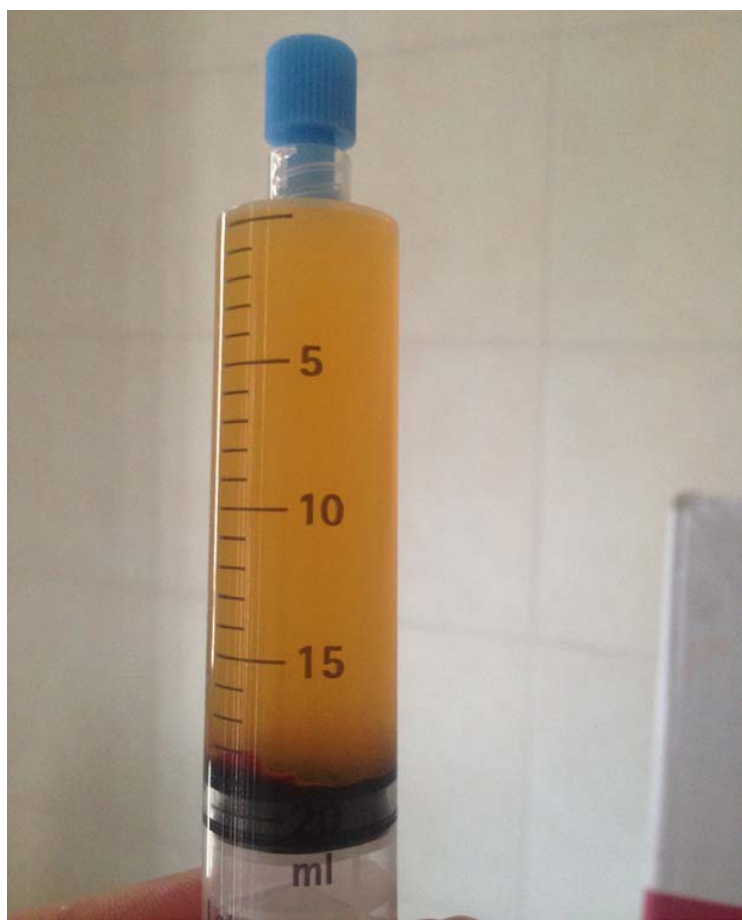

Fig. 3. Result of the 2 nd centrifugation - Hard Spin. PRP in $1 / 3$ basal volume

This method assumes observance of aseptic conditions when performing the procedure only in the connector parts of the syringes and the disc reflux concerning blood and its components and does not require general sterility, which is sterilization of the centrifuged part of the rotor (rotor cups) and can be performed in the operating unit clinical laboratory. PRP suitable for use in non-sterile syringes may be aspirated with a sterile syringe with a variable needle and, after changing the needle, used under aseptic operating conditions. In order to monitor the effectiveness of concentration and determine the direct quality of PRP, it is advisable to determine the platelet count and hemoglobin level using standard laboratory analyzers used in clinical laboratory practice. We offer for use semi-automatic analyzers. In order to achieve an appropriate quality of centrifugation and fractionation, an important criterion is to preserve the maximum number of platelets in the final substrate. Another criterion is the minimum level of hemoglobin (up to $5 \mathrm{~g} / \mathrm{l}$ ), which is an indicator of sufficient precision of the detachment of the buffer layer at the stage of the 
first centrifugation. For example: with a patient's hemoglobin level of $130 \mathrm{~g} / \mathrm{l}$ and a platelet level of 250 thousand/ $\mu \mathrm{l}$ at a concentration of 5 times (that is, about $15 \mathrm{ml}$ of PRP obtained from $72 \mathrm{ml}$ of whole blood collected from the patient) analyzer in 1 million $\mu 1$. A sufficient quantitative indicator of the qualitative detachment of the buffer layer will be the level of hemoglobin of the final substrate - PRP at a level of up to $5 \mathrm{~g} / \mathrm{l}$.

The results of a quantitative assessment of the concentration of platelets in the whole blood of the subjects as well as in the PRP substrate harvesting technique described above are presented. The results are shown in Table $\mathbf{1 .}$

Table 1

Assessment of platelet levels of whole blood and the resulting PRP study group

\begin{tabular}{ccccc}
\hline The number of studied & $\begin{array}{c}\text { Initial average platelet } \\
\text { level of whole blood, } \\
\text { thousand in } \mathbf{~ m l}\end{array}$ & Centrifuging mode & $\begin{array}{c}\text { The average number } \\
\text { of platelets in PRP } \\
\text { thousand in } \mathbf{~ m l}\end{array}$ & Multiplication factor \\
\hline 10 & $\mathbf{2 2 7 . 0} \pm \mathbf{5 7 . 0}$ & $\begin{array}{c}160 \mathrm{~g} \times 10 \mathrm{~min}+ \\
+250 \mathrm{~g} \times 15 \mathrm{~min}\end{array}$ & $\mathbf{9 4 5 . 0} \pm \mathbf{2 7 9 . 0}$ & 4.16
\end{tabular}

\section{Discussion}

The proposed protocol for obtaining plasma enriched with platelets is characterized by the use of consumables widely available in clinical practice and makes it possible to be adapted to many types of centrifuges and their rotors, which makes it possible to consider the proposed technology to be fundamentally more accessible in comparison with the analogues on the market.

For centrifuging, most rotor centrifugal centrifuges are adapted, equipped with rotary cups rotors, which are widely used in clinical and laboratory practice. As a consumable, the method requires an appropriate plasma-rich platelet volume that requires a number of syringes with a LuerLock attachment. For example, to get $16 \mathrm{ml}$ of PRP you need 7 syringes 20.0. A combi-stopper plug is used for each syringe for each centrifugation step. With careful performance and observance of aseptic conditions in the connectors during the procedure, it is possible to use one three-way valve disc fixes. The ACD-A solution is used as an anticoagulant available and does not require special storage conditions and can be used in various packing volumes, provided it is stored under aseptic conditions during storage and used.

A solution of $\mathrm{CaCl}_{2} 10 \%$, which is used as an activator is widely available [19]. In addition, the proposed method allows a differentiated approach to the procedure given the required volume of PRP (from $2 \mathrm{ml}$ to $16 \mathrm{ml}$ per procedure), which is convenient in medical practice considering the wide range of tasks for the application of the substrate. The possibility of obtaining significant amounts of PRP in comparison with standard methods is an important improvement that allows us to recommend a technique for use in general surgical practice, which often requires a significant amount of PRP-10 $\mathrm{ml}$ or more.

\section{Conclusions}

1. The proposed method of separation of whole blood with the release of the plasma rich with platelets demonstrates high efficiency, which corresponds to the level of increasing the number of platelets in reducing the volume at the level of the best ready-made solutions.

2. The equipment is economical and does not require highly specialized equipment and consumables.

3. The proposed technique provides a wide choice to the performer in the received volume of the substrate.

\section{References}

[1] Dhurat, R., Sukesh, M. (2014). Principles and methods of preparation of platelet-rich plasma: A review and author's perspective. Journal of Cutaneous and Aesthetic Surgery, 7 (4), 189. doi: https://doi.org/ 10.4103/0974-2077.150734 
[2] Martinez-Zapata, M. J., Martí-Carvajal, A. J., Solà, I., Expósito, J. A., Bolíbar, I., Rodríguez, L. et. al. (2016). Autologous platelet-rich plasma for treating chronic wounds. Cochrane Database of Systematic Reviews. doi: https://doi.org/10.1002/14651858.cd006899.pub3

[3] Sister, D. (2016). PRP: the new frontier in regenerative medicine and aesthetic medicine. Firence, 158.

[4] Fareed, W. M., Tandon, P., Ahmad Z, E., Gazal, G., Hussain Khan, A., Zaidi, T. et. al. (2017). Efficacy of Blood and Its Products-boon for Oral Surgeons: Review. Journal of Universal Surgery, 05 (01). doi: https://doi.org/10.21767/2254-6758.100071

[5] Joshi Jubert, N., Rodríguez, L., Reverté-Vinaixa, M. M., Navarro, A. (2017). Platelet-Rich Plasma Injections for Advanced Knee Osteoarthritis: A Prospective, Randomized, Double-Blinded Clinical Trial. Orthopaedic Journal of Sports Medicine, 5 (2), 232596711668938. doi: https://doi.org/10.1177/2325967116689386

[6] Parizzi, N. G., Rubini, O. Á., Almeida, S. H. M. de, Ireno, L. C., Tashiro, R. M., Carvalho, V. H. T. de. (2017). Effect of platelet-rich plasma on polypropylene meshes implanted in the rabbit vagina: histological analysis. International Brazj Urol, 43 (4), 746-752. doi: https://doi.org/10.1590/s1677-5538.ibju.2016.0177

[7] Dönmez, M. İ., İnci, K., Zeybek, N. D., Doğan, H. S., Ergen, A. (2016). The Early Histological Effects of Intravesical Instillation of Platelet-Rich Plasma in Cystitis Models. International Neurourology Journal, 20 (3), 188-196. doi: https://doi.org/10.5213/inj.1632548.274

[8] McCarrel, T. M., Minas, T., Fortier, L. A. (2012). Optimization of Leukocyte Concentration in Platelet-Rich Plasma for the Treatment of Tendinopathy. The Journal of Bone \& Joint Surgery, 94 (19), e143. doi: https://doi.org/10.2106/jbjs.1.00019

[9] Bausset, O., Giraudo, L., Veran, J., Magalon, J., Coudreuse, J.-M., Magalon, G. et. al. (2012). Formulation and Storage of Platelet-Rich Plasma Homemade Product. BioResearch Open Access, 1 (3), 115123. doi: https://doi.org/10.1089/biores.2012.0225

[10] Dhurat, R., Sukesh, M. (2014). Principles and methods of preparation of platelet-rich plasma: A review and author's perspective. Journal of Cutaneous and Aesthetic Surgery, 7 (4), 189. doi: https:// doi.org/10.4103/0974-2077.150734

[11] Fitzpatrick, J., Bulsara, M. K., McCrory, P. R., Richardson, M. D., Zheng, M. H. (2017). Analysis of Platelet-Rich Plasma Extraction. Orthopaedic Journal of Sports Medicine, 5 (1), 232596711667527. doi: https://doi.org/10.1177/2325967116675272

[12] Kushida, S., Kakudo, N., Morimoto, N., Hara, T., Ogawa, T., Mitsui, T., Kusumoto, K. (2014). Platelet and growth factor concentrations in activated platelet-rich plasma: a comparison of seven commercial separation systems. Journal of Artificial Organs, 17 (2), 186-192. doi: https://doi.org/10.1007/s10047-014-0761-5

[13] Kececi, Y., Ozsu, S., Bilgir, O. (2014). A cost-effective method for obtaining standard platelet-rich plasma. Wounds, 26, 232-238.

[14] Foster, T. E., Puskas, B. L., Mandelbaum, B. R., Gerhardt, M. B., Rodeo, S. A. (2009). Platelet-Rich Plasma. The American Journal of Sports Medicine, 37 (11), 2259-2272. doi: https://doi.org/10.1177/ 0363546509349921

[15] Perez, A. G. M., Lana, J. F. S. D., Rodrigues, A. A., Luzo, A. C. M., Belangero, W. D., Santana, M. H. A. (2014). Relevant Aspects of Centrifugation Step in the Preparation of Platelet-Rich Plasma. ISRN Hematology, 2014, 1-8. doi: https://doi.org/10.1155/2014/176060

[16] Degen, R. M., Bernard, J. A., Oliver, K. S., Dines, J. S. (2016). Commercial Separation Systems Designed for Preparation of Platelet-Rich Plasma Yield Differences in Cellular Composition. HSS Journal ${ }^{\circledR}$, 13 (1), 75-80. doi: https://doi.org/10.1007/s11420-016-9519-3

[17] Magalon, J., Bausset, O., Serratrice, N., Giraudo, L., Aboudou, H., Veran, J. et. al. (2014). Characterization and Comparison of 5 Platelet-Rich Plasma Preparations in a Single-Donor Model. Arthroscopy: The Journal of Arthroscopic \& Related Surgery, 30 (5), 629-638. doi: https://doi.org/10.1016/ j.arthro.2014.02.020

[18] Yin, W., Xu, H., Sheng, J., Zhu, Z., Jin, D., Hsu, P. et. al. (2017). Optimization of pure platelet-rich plasma preparation: A comparative study of pure platelet-rich plasma obtained using different centrifugal conditions in a single-donor model. Experimental and Therapeutic Medicine, 14 (3), 2060-2070. doi: https://doi.org/10.3892/etm.2017.4726

[19] Cavallo, C., Roffi, A., Grigolo, B., Mariani, E., Pratelli, L., Merli, G. et. al. (2016). Platelet-Rich Plasma: The Choice of Activation Method Affects the Release of Bioactive Molecules. BioMed Research International, 2016, 1-7. doi: https://doi.org/10.1155/2016/6591717 